\title{
FLUIDASSEM - A NEW METHOD OF FLUIDIC-BASED ASSEMBLY WITH SURFACE TENSION
}

\author{
N. Boufercha ${ }^{1}$, J. Sägebarth ${ }^{1}$, M. Burgard ${ }^{1}$, N. Othman ${ }^{2}$, \\ D. Schlenker ${ }^{2}$, W. Schäfer ${ }^{2}$, H. Sandmaier ${ }^{1}$ \\ ${ }^{1}$ Universität Stuttgart, IFF / MST, Nobelstr. 12, 70569 Stuttgart, Germany \\ www.iff.uni.stuttgart.de \\ ${ }^{2}$ Fraunhofer, IPA, Nobelstr. 12, 70569 Stuttgart, Germany \\ www.ipa.fraunhofer.de
}

\section{Introduction}

Regarding electronic components the reduction of costs will be a challenging goal in the next few years. The use of polymer electronics will not help to minimise this problem. Cheap products made of silicon are here to stay for a while.

The latest roadmap of the VDI/VDE-IT (see MINAM-Roadmap: www.micronanomanufacturing.eu) indicates that the size of assembled silicon chips must dramatically be reduced. The size of these chips will be less than $500 \mu \mathrm{m} \mathrm{x}$ $500 \mu \mathrm{m}$ and will have a thickness lower than 50 to $70 \mu \mathrm{m}$. The reduction of the silicon material must be in parallel to the reduction of the costs for the manufacturing process.

An indicator for this trend is also shown in Figure 1. For example the expected world market share for RFID in 2010 will be about 11.7 billion USD (Frost \& Sullivan 2004). This can only happen if the overall costs (microchip and manufacturing) decrease. The conventional manufacturing methods for microchips with conventional die-bonders have also reached their limit concerning accuracy of positioning, throughput and handling of smallest microchips and micro components. The expected requirements effected by downscaling make it necessary to invent new innovative technologies for production like FluidAssem.

The main topic in the next few years will be the development of processes which have the capability to use self assembly methods ([3], [10], [6]). The presented paper investigates methods for processes allowing self assembly of microchips. Additionally the vision is traced to establish an assembly process without using pick and place methods as used in die-bonder processes today. A new concept FluidAssem was developed to establish methods for reaching this challenging goal of a safe assembly process for the future. 


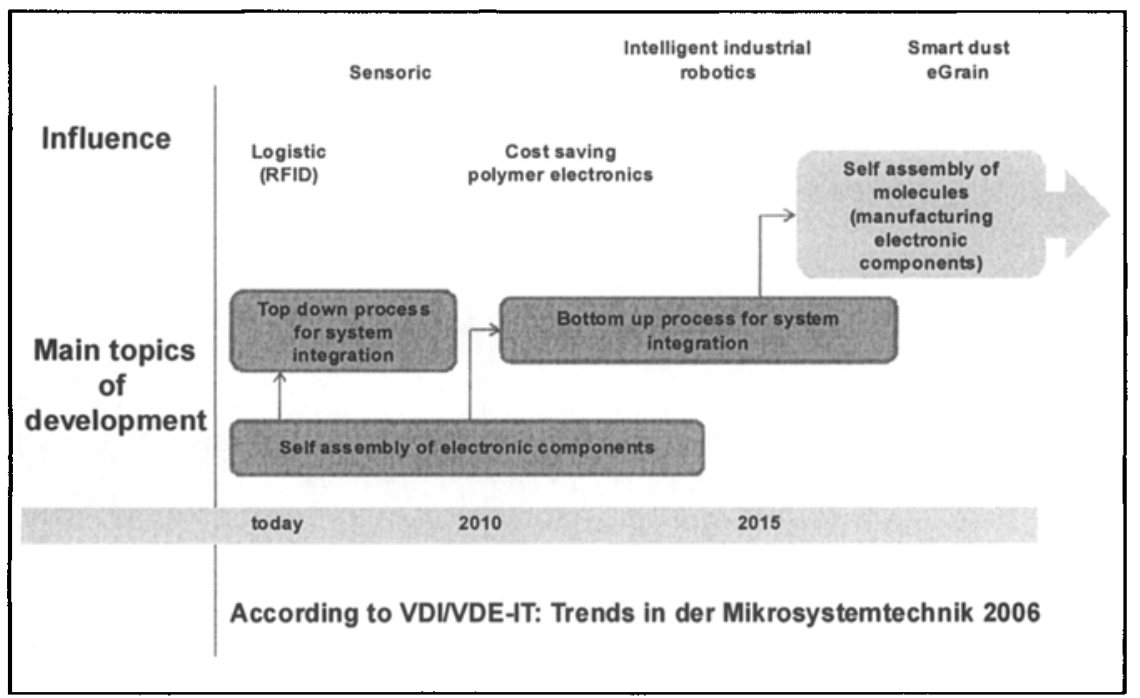

Fig. 1. Roadmap microsystems

\section{The Micro Cosmos}

It is well known that in the micro cosmos surface forces prevail against volume forces. Why not use these forces for transporting and assembling small chips and components? The surface forces are high enough to do all the things needed for the assembly process of micro components!

To move droplets on a substrate, various methods can be used. The most famous technique is the use of electro wetting. An electric field partially changes the contact angle on one side of the droplet. The droplet moves in the direction of the smaller contact angle because the surface tension of the droplet wants to minimise the surface energy. This motion stops if the droplet has reached a position with a local minimum of energy. In this case the movement of the droplet depends directly on the contact angles realised by an electric field.

Positioning of droplets is also possible by changing only the surface properties ([14], [13], [11], [5], [2]). Therefore, the surface of a substrate must be structured with hydrophilic and hydrophobic areas [7]. These structures are usually a combination of coating with additional micro- and nanostructures to get super hydrophilic or super hydrophobic areas. An area structured this way allows guiding droplets along a specified way.

If a single droplet can move from one to another position, this can also be done with small chips "swimming" on those droplets. With a skillfully structured surface on the substrate and an intelligent use of the surface tension of the fluid, a self alignment process for micro components can be achieved. 
In Figure 2 a principle method for fluidic assembly is given. In a first step a large droplet (diameter about $200 \mu \mathrm{m}$ ) is placed in the centre of the substrate. This droplet has the job of catching the falling microchip and placing this microchip in the correct position. Smaller droplets are used to contact the microchip in the final position. Special micro and nano structured regions are used to hold the drops in the starting position on the substrate.

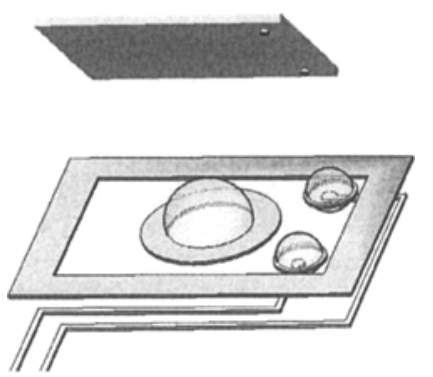

Fig. 2. Principles of fluidic assembly

To achieve a fluidic assembly system, the realised surface properties ([9]), the structures on the substrate and the whole process should first be simulated and optimised.

The whole process can be analysed via decomposition. The steps in detail are as follows:

- placing the droplets for catching and contacting the microchip on the correct starting position on the substrate

- putting the micro chip on the top of the catching droplet (middle one) without mismatching the position (vertical alignment)

- adding a mismatch between starting and end position of the chip (lateral

- alignment)

- pinning effect (contact angle hysteresis)

- checking the accurateness of the assembly process

This decomposition of the whole process allows an easy and effective estimation of the assembly process. It can also be used to calculate an upper limit of the assembly process.

\section{Simulation Process}

The simulation of the dynamic behaviour of a droplet on a structured substrate surrounded by a gaseous environment is done with multiphase models which take 
wetted walls into account. These models must have the capability to track the fluidfluid interface and to describe changes in physical properties such as density, viscosity, contact angle etc. The non-conservative level set method [4] is used as the computation method for solving these problems. The level set method is based on continuum approach in order to represent surface tension and local curvature at the interface as a body force. This allows capturing any topological changes due to changes in surface tensions. In this method the interface between two phases is represented by a smooth function, called level set function $\Phi(r, t)$. The level set function is always positive in the continuous phase and is always negative in the dispersed phase. The interface is implicitly represented by the points where the level set function is zero. From such a representation of an interface we can calculate the motion of the free surface by advection of the level set function:

$$
\frac{\partial \Phi}{\partial t}+\mathbf{u} \nabla \Phi=\gamma \nabla[\epsilon \nabla \Phi-\Phi(1-\Phi) \cdot \mathbf{n}]
$$

The governing equations of motion for the incompressible isothermal flow can be written in terms of the Navier-Stokes equation which is the equation for the fluid velocity $u$ and pressure $p$ [12]. The Navier-Stokes equation describes the balance of force densities $\mathrm{f}_{\mathrm{type}}$ acting on fluid elements.

$$
\varrho\left[\frac{\partial \mathbf{u}}{\partial t}+(\mathbf{u} \nabla) \mathbf{u}\right]=f_{\text {press }}+f_{\text {frict }}+f_{v o l}
$$

The body force $\mathrm{f}_{\mathrm{vol}}$ includes gravitational force and the surface tension term due to the level set treatment of interfacial stresses. The $f_{\text {vol }}$ term is represented by the two components

$$
f_{k: o l x}=\sigma \cdot k \cdot \frac{\partial \Phi}{\partial x} \cdot \delta(\Phi) \quad f_{\text {woly }}=\sigma \cdot k \cdot \frac{\partial \Phi}{\partial y} \cdot \delta(\Phi)+\rho \cdot g
$$

The surface tension term at the interface which is determined by the position of the zero level set is treated by the delta function $\delta(\Phi)$. The curvature $\kappa$ of the fluidic interface is represented in terms of level set function

$$
\text { s: }=\nabla \mathbf{n}
$$

The unit vector $\mathrm{n}$ on the interface points from dispersed phase to continuous phase. In terms of the level set function the unit vector can be described as

$\mathbf{n}=\frac{\nabla \Phi}{|\nabla \Phi|}$

The change in physical properties is described by the Heavyside function which is represented in terms of level set function

$$
H(\Phi<0)=0 \quad H(\Phi=0)=\frac{1}{2} \quad H(\Phi>0)=1
$$


Schäfer, H. Sandmaier

Density and viscosity which are constant in each fluid are represented in terms of Heavyside function as

$$
\rho=H(\Phi)+\frac{\rho_{\text {discret }}}{\rho_{\text {continous }}}(1-H(\Phi)) \quad \eta=H(\Phi)+\frac{\eta_{\text {discret }}}{\eta_{\text {continous }}}(1-H(\Phi))
$$

The finite element method is used for modeling and simulation. The set of equations are solved by using "COMSOL Multiphysics" which allows also the coupling of different physical models [15].

\section{Self assembly Process}

For the analysis of the assembly process the model will be simplified. In the following the droplets for contacting the microchip will not be taken into account. So the first process step will be the placement of a droplet (diameter about $200 \mu \mathrm{m}$ on the substrate. This droplet has the job of catching the microchip.
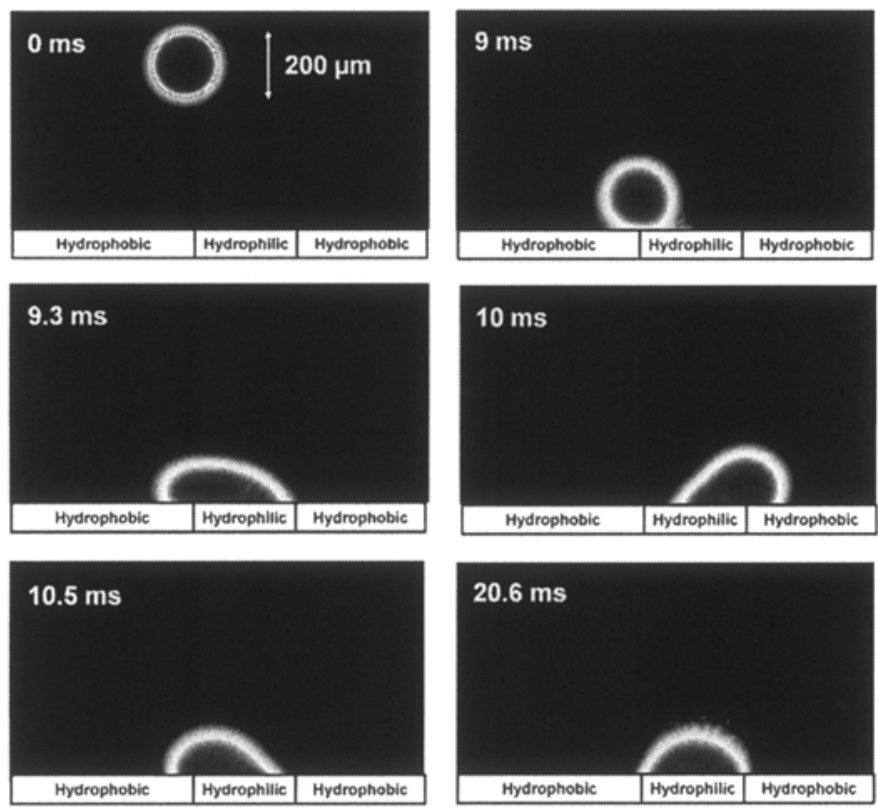

Fig.3. Simulation of the self alignment of a fluid 
Tension

The droplet in Figure 3 will fall on the functionalised surface. In the example shown in Figure 3 the droplet hits the surface at the interface between the hydrophilic and hydrophobic area. This is to simulate the limited exactness by dispensing the droplets for catching the microchips. After the droplet hits the substrate, it moves in the direction of the hydrophilic surface (picture of the simulation at $9 \mathrm{~ms}$ in Figure 3). Reaching the right hydrophobic area, the droplet will overshoot the hydrophobic area because of its mass inertia (time $=10 \mathrm{~ms}$ ). The acceleration of the droplet is again in direction of the hydrophilic array. The droplet will slow down and move back into the other direction. After some oscillations the droplet will reach a stable position. In about $20 \mathrm{~ms}$ the droplet is placed fully on the hydrophilic area and is ready for catching a microchip from a dispenser.

Beside the time needed to get a stable drop, it is important to know the size of the hydrophobic ring around the hydrophilic area to fix the droplet in the correct starting position (see figure 2). Some simple considerations with undamped oscillations show that the overshoot of the droplet will be in the same range as the deviation from the centre where the drop is dispensed.

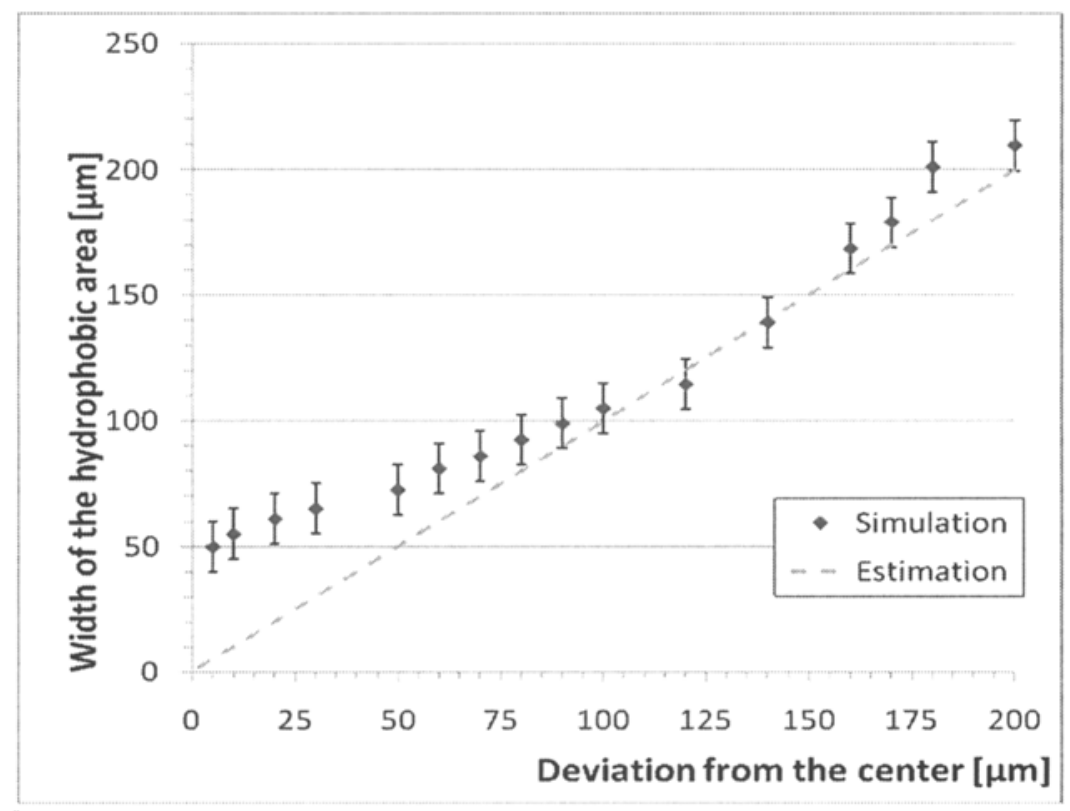

Fig. 4. Size of the hydrophobic barrier 
In Figure 4 this estimation is presented as a dotted line. With a detailed and very time consuming simulation of a two phase fluidic system, this simple consideration is relatively good for a large deviation from the centre, but for small deviations the size of the hydrophobic ring will not become zero. The minimum size of this ring will be about $50 \mu \mathrm{m}$ for the parameter used in the simulation (contact angle hydrophilic: $60^{\circ}$, contact angle hydrophobic: $120^{\circ}$, surface tension $72.5 \mathrm{mN} / \mathrm{m}$, viscosity, ...). With an estimated accuracy of $50 \mu \mathrm{m}$ by dispensing the droplet, the hydrophobic barrier must be about $80 \mu \mathrm{m}$ (see figure 4).

After the droplet is placed on the substrate, the microchip is set on the top of the droplet.

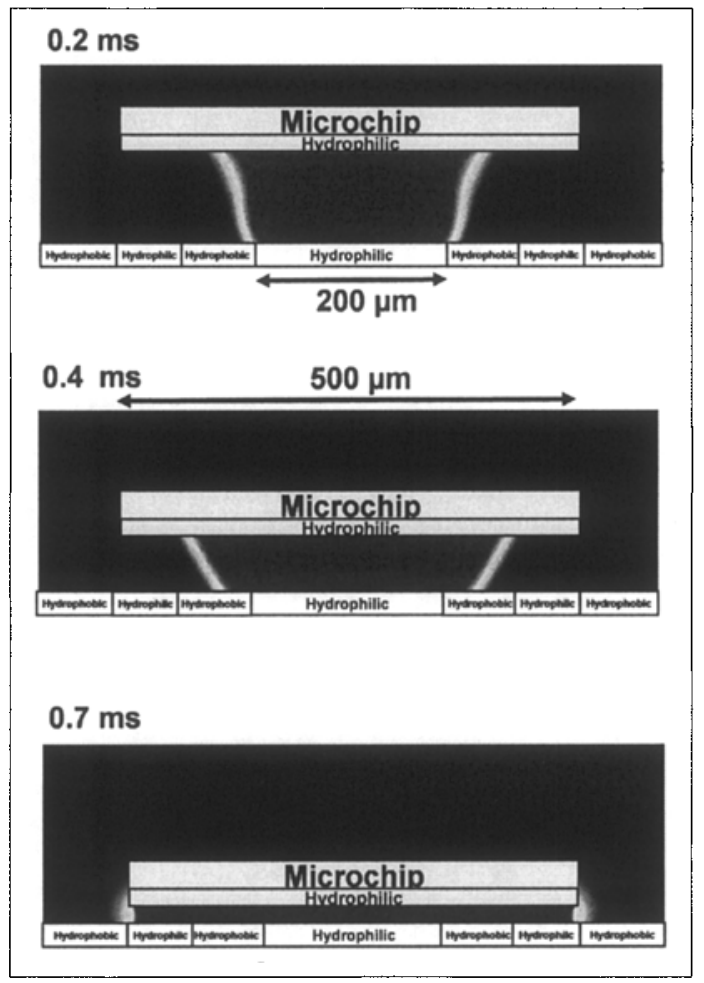

Fig. 5. Simulation of the self alignment capability of a microchip

As described before this phase of the assembly process is split into two parts. In the first part only the vertical lowering of the microchip is investigated. The horizontal alignment of the microchip is considered in the second part.

In the first part there is only a vertical and no horizontal mismatching of the chip. Figure 5 at $t=0.2 \mathrm{~ms}$ shows the situation when the microchip reaches the top of the droplet placed on the substrate. 
If the hydrophilic microchip (yellow) touches the fluidic interface of the droplet, the fluid creeps along the surface of the microchip. The high surface tension of the changed shape of the droplet overrides the hydrophobic area (white). A new fluidic interface between the chip and the substrate is set up (figure 5 after $0.7 \mathrm{~ms}$ ).

In the second part the lateral (horizontal) alignment function is investigated. For this alignment the surface tension on the right and left side of the chip tries to minimize the surface energy. The fluidic segment shell allows an easy horizontal movement and the microchip will be automatically horizontally aligned on the correct final position for assembly.

An analytical estimation of the time for this horizontal alignment is

$$
t \approx l \cdot \sqrt{\frac{g \cdot d}{\sigma} \cdot y}
$$

1 is the edge length and $d$ is the thickness of the microchip, $y$ is the relative mismatching of positioning of the microchip.

Figure 6 shows the relation between the time needed for the horizontal adjustment and the deviation from the final position based on the above mentioned estimation for the alignment of a microchip.

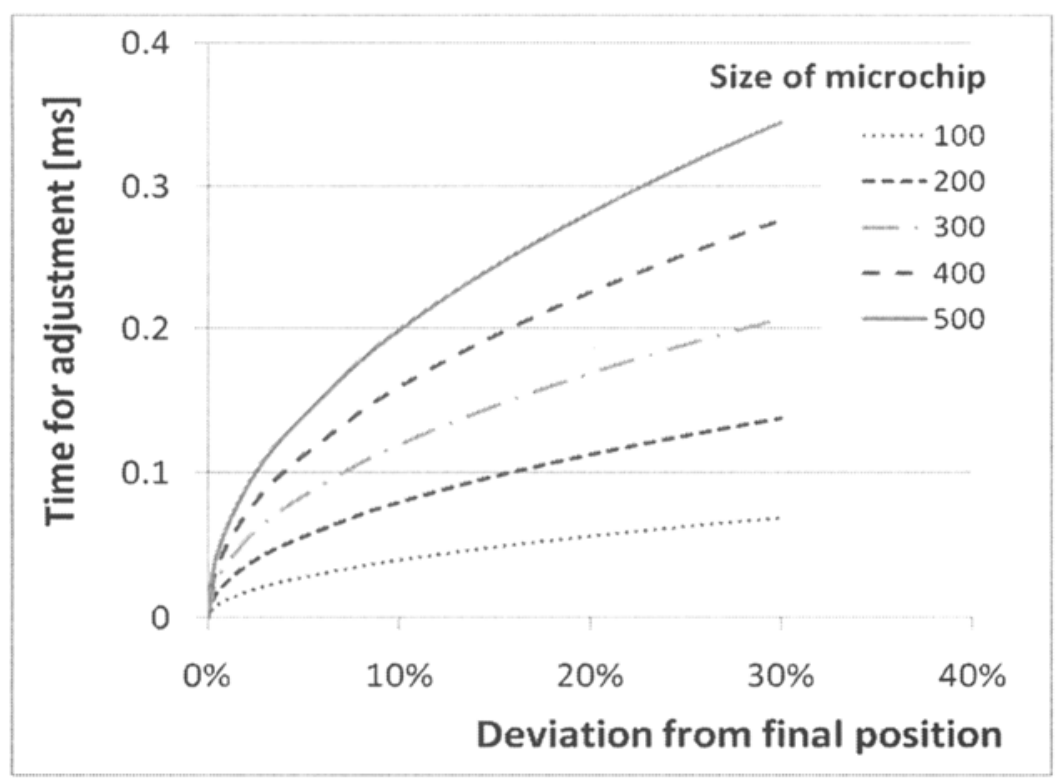

Fig.6. Alignment of the microchip 
Schäfer, H. Sandmaier

The analytical estimation in Figure 6 shows that a microchip with a size of $500 \mu \mathrm{m}$ and a deviation of up to 30 percent from the final position needs less than $0.35 \mathrm{~ms}$ for horizontal adjustment. The catching of the microchip and filling of the area between microchip and substrate with the fluid is calculated by the simulation. The numerically calculated time for the vertical adjustment is about $0.7 \mathrm{~ms}$ (see figure 5).

The horizontal and vertical adjustments are taking place simultaneously. The total time for the alignment process will be less than one millisecond.

After the microchip has reached the final position, it can be fixed and contacted on the substrate.

\section{Accuracy of Assembly}

The whole assembly process is driven by the surface tension. In principle this surface tension builds up an interface with the lowest possible energy. To get the best results the hysteresis of the contact angle should be zero. The improvement of the hydrophilic and hydrophobic areas on the surface of the substrate needs a nanostructured interface. Unfortunately the nanostructuring leads to a stronger pinning effect and also to a bigger hysteresis $\gamma$ of the contact angel. According to Figure 7 this pinning effect prevents a proper alignment of the microchip.

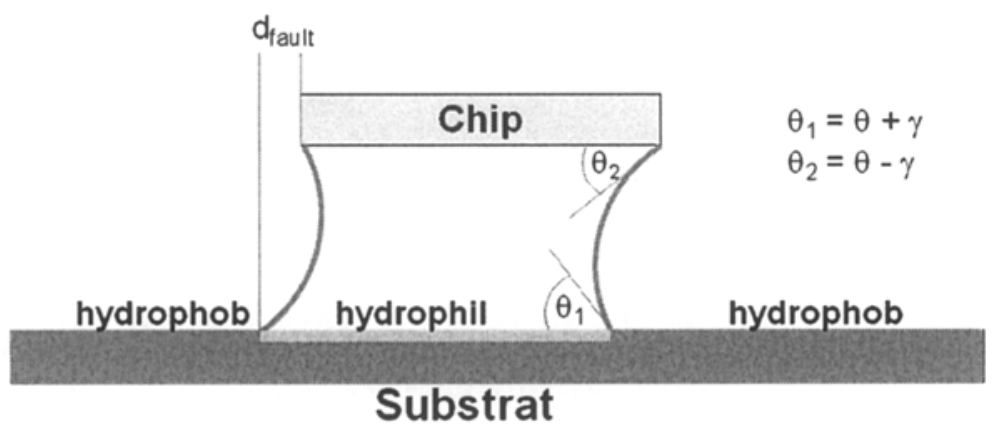

Fig. 7. Exactness of positioning (model)

Assuming that the vertical size (thickness) of the fluid between the substrate and the microchip will be very small, an approximation of the form of the interface by a sphere structure is appropriate enough. The biggest error of the alignment will come up if on the top (side of microchip) the contact angle $\theta$ will be reduced by the hysteresis angle $\gamma$ and on the bottom (side of substrate) the contact angle $\theta$ will be increased by the hysteresis angle. In this case an approximation of the position fault $d_{\text {fault }}$ depending of the drop size $d_{\text {drop }}$ and the diameter of the microchips $l_{\text {chip }}$ can be given 


$$
d_{\text {faut }}=\frac{1}{6} \cdot \pi \cdot \frac{d_{d r o p}^{3}}{l_{c h i p}^{2}} \cdot \tan \gamma
$$

The simulation (figure 5) shows a microchip with a diameter of about $500 \mu \mathrm{m}$ which is caught by a droplet with a size of about $200 \mu \mathrm{m}$. The hysteresis $\gamma$ of the contact angle is about $20^{\circ}$. In this case the error of positioning of the microchip is smaller than $10 \mu \mathrm{m}$.

\section{Summary}

This paper shows a new assembly method called FluidAssem for the assembly of small chips and other micro components. This new assembly process uses the downscaling effect where surface forces prevail over volume forces. The basis of this process is a special substrate with hydrophobic and hydrophilic structured areas. These structures allow the guidance of micro components via microfluidic forces to the exact assembly positions.

In the assembly process the micro components can be supplied to the substrate via a microfluidic dispensing process ([1], [8]). The fluidic system aligns the micro components on the correct positioning by the use of surface tensions. In this final position the micro component will be fixed and connected. The predicted exactness of the positioning of the microchips will fulfill the requested requirements (exacter than $10 \mu \mathrm{m}$ ) for the assembly process. The simulation shows that the time for placing a droplet on a substrate will be about $20 \mathrm{~ms}$ and the time for aligning the micro component will be about $1 \mathrm{~ms}$. Compared to die-bonders used today, a higher throughput of assembled chips per hour can be expected.

With the resign of kinematic components the assembly process will have the capability to handle smallest microchips and micro components.

\section{References}

1. H. Sandmaier, R. Zengerle, B. de Heij, C. Steinert. A Tunable and Highly-Parallel Picoliter-Dispenser Based on Direct Liquid Displacement. In MEMS 2002, Las Vegas, USA, 2002.

2. J. Berthier and P. Silberzahn. Microfluidics for Biotechnology. Artech House, 2006.

3. J. Fang and K. F. Böhringer. High yield batch packaging of Micro Devices with uniquely orienting Self-Assembly. In IEEE International Electronic Devices Meeting, 2005.

4. M. Fietz. Numerische Simulation von Oberflächeninstabilitäten in Zweiphasenströmungen mit Hilfe einer Level-Set Methode. PhD thesis, RWTH Aachen, 2003.

5. T. Franke and A. Wixforth. Das Labor auf dem Chip. Physik unserer Zeit, 38:8894, 2007.

6. G. Engelmann, H. Reichl, M. Hutter, H. Oppermann. High Precision Passive Alignment Flip Chip Assembly Using Self-alignment and Micro-mechanical Stops. In Electronics Packaging technology Conference, 2004. 
Schäfer, H. Sandmaier

7. F. Mugele. Liquids in Contact with Solids: Nanotribology and Micro-fluidics. PhD thesis, Universität Ulm - Abteilung Angewandte Physik, 2004.

8. C. P. Steinert, H. Sandmaier, S. Messner, B. de Heij, M. Daup, R. Zengerle, O. Gutmann, R. Niekrawietz. Droplet Release in a Highly Parallel Pressure Driven Nanoliter Dispenser. In Transducers '03, Boston, 2003

9. S. Pal, D. Roccatano, H. Weiss, H. Keller and F. Müller-Plathe. Molecular Dynamics Simulation of water near nanostructured hydrophobic surfaces: interfacial energies. ChemPhysChem, 6:1641-1649, 2005

10. J. S. Smith. High density, low parasitic direct integration by Fluidic Self Assembly (FSA). In IEEE International Electronic Devices Meeting, 2000.

11. A. Torkkeli. Droplet microfluidic on a planar surface. PhD thesis, VTT Publications 504, 2003.

12. E. Truckenbrodt. Fluidmechanik, Band I, Grundlagen und elementare Strömungsvorgänge dichtebeständiger Fluide, 4. Auflage. Springer-Verlag, Berlin, 1996.

13. F. Exl und J. Kindersberger. Messung von Tropfenrandwinkeln auf Isolierstoffoberflächen. In ETG-Fachbericht 97, VDE-Verlag GmbH Berlin Offenbach, S. 67-72, 2004.

14. L. Zhu, Y. Feng, X. Ye and Z. Zhou. Tuning wettability and getting superhydrophobic surfaces by controlling surface roughness with well-designed microstructures. Sensors and Actuators, A 130-131:595-600, 2006.

15. W. B. J. Zimmerman. Multiphysics Modelling with finite Element Methods. World Scientific Publishing Co.Pte.Ltd. , 2006. 